\title{
Proposta de escore preditor de desfechos para abdome agudo.
}

\section{Outcomes prediction score for acute abdomen: a proposal.}

Felipe Antônio Cacciatori ${ }^{1,2}$; $;$ Arthur Dajori Ronchi ${ }^{1,3}$; Sérgio Emerson Sasso ${ }^{1}$

R E S U M O

\begin{abstract}
Objetivo: estabelecer a correlação entre dados objetivos coletados na avaliação inicial e os desfechos nos casos de dor abdominal aguda. Métodos: estudo retrospectivo, de caso controle, em que foram revisados prontuários de pacientes atendidos em um serviço de urgência e emergência no ano de 2016, diagnosticados com dor abdominal e pélvica. Resultados: de 2.352 prontuários avaliados, 330 foram considerados válidos para o estudo. Destes pacientes, 235 (71,2\%) receberam alta e os $95(28,8 \%)$ restantes foram internados, submetidos à cirurgia ou morreram. A análise estatística demonstrou que sexo masculino, idade $\geq 50$ anos, temperatura axilar $>37,3^{\circ} \mathrm{C}$, anemia, leucocitose $>10.000 / \mathrm{mm}^{3}$, neutrocitose acima de $7.700 / \mathrm{mm}^{3}$, linfopenia $<2.000 / \mathrm{mm}^{3}$ e hiperamilasemia são variáveis isoladamente associadas a pior desfecho. Conclusão: a presença de três ou mais das variáveis avaliadas aumenta fortemente a chance de um paciente sofrer os desfechos de cirurgia ou morte, sendo a chance de óbito tanto maior quanto maior o número de variáveis presentes.
\end{abstract}

Descritores: Dor Abdominal. Abdome Agudo. Pontuação de Propensão. Centro Cirúrgico Hospitalar.

\section{INTRODUÇÃO}

bdome agudo pode ser definido como a
presença de dor e sensibilidade abdominal ${ }^{1}$ de etiologia não traumática, com duração máxima de cinco dias², e que pode ser causado por mais de 42 enfermidades distintas ${ }^{3}$, muitas das quais de tratamento cirúrgico. O objetivo primário nestes casos é o diagnóstico precoce e correto da etiologia, para, então, dar início ao tratamento adequado 4 . Entretanto, cerca de $40 \%$ dos pacientes que procuram assistência médica com queixas de dor abdominal permanecem sem diagnóstico ${ }^{5}$.

Remanesce dos tempos de Hipócrates, sem jamais ter perdido a importância, o pensamento de que o exame clínico deve ser soberano. Contudo, sabe-se também que apenas $43 \%$ a 59\% dos diagnósticos baseados na anamnese e exame físico são acertados ${ }^{2}$, pois é frequente a normalidade dos sinais vitais, a despeito de uma doença grave, sobretudo em pacientes idosos ou imunocomprometidos. Sequer a intensidade da dor abdominal guarda alguma relação com a gravidade da doença ${ }^{6}$.
Neste cenário, o bom uso dos métodos complementares pode auxiliar a aumentar o índice de diagnósticos corretos ou, ao menos, indicar os pacientes que necessitam de investigação ampliada.

A importância do tema fica sobremaneira evidenciada quando se leva em conta que, no Brasil, somente no mês de janeiro de 2018, foram registradas no DATASUS 3.419 internações por dor abdominal e pélvica (CID10 R10), o que resultou em 18.553 dias acumulados de internação. Na mesma amostra, foram registrados 329 óbitos, correspondendo a uma taxa de letalidade de 9,62\% ${ }^{7}$.

O desenvolvimento de algoritmos ou escores diagnósticos que aumentem a acurácia nos casos de abdome agudo, até o presente inexistentes ${ }^{2}$, poderia, em última análise, contribuir para a redução da morbimortalidade por doenças abdominais. O primeiro passo rumo a esse objetivo inclui o estabelecimento da correlação entre os dados objetivos obtidos na avaliação inicial, incluindo os exames complementares mínimos, com o desfecho de pacientes que se apresentam com dor abdominal aguda no serviço de urgência e emergência.

1 - Universidade do Extremo Sul Catarinense (UNESC), Curso de Medicina, Criciúma, SC, Brasil. 2 - Hospital São José, Serviço de Cirurgia Geral, Criciúma, SC, Brasil. 3 - Hospital São José, Serviço de Anestesiologia, Criciúma, SC, Brasil. 
O objetivo deste estudo foi estabelecer uma correlação entre dados objetivos coletados na avaliação inicial dos pacientes atendidos por dor abdominal aguda e os seus desfechos clínicos.

\section{MÉTODOS}

Estudo retrospectivo e de caso-controle composto por pacientes atendidos no serviço de pronto socorro de um hospital de ensino de média e alta complexidade, localizado em um município do sul de Santa Catarina, que serve a uma população de 994 mil habitantes e que, à época, realizava cerca de 130 mil atendimentos/ano.

Após aprovação pelos Comitês de Ética em Pesquisa da Universidade do Extremo Sul Catarinense (UNESC) e do hospital, com pareceres consubstanciados de número 2.253 .110 e 2.278.239, respectivamente, por meio do sistema Plataforma Brasil, foram levantados todos os atendimentos classificados com CID10 R10 (dor abdominal e pélvica) e realizados no ano de 2016, totalizando 4.575 lançamentos. Os atendimentos foram randomizados utilizando o comando "Aleatório" no software Microsoft Excel 2016. Os pesquisadores, então, obtiveram acesso aos prontuários armazenados no sistema hospitalar Philips TASY, de onde os dados foram coletados, consecutivamente, até obter-se a amostra final de 330 atendimentos válidos, com 2.022 exclusões, todas em virtude de prontuário incompleto. Não foram identificados atendimentos repetidos na amostra de prontuários válidos. A figura 1 sumariza as etapas do presente estudo.

A tabulação dos dados deu-se no software Microsoft Excel e dois grupos foram concebidos: o grupo Controle, composto pelos pacientes que receberam alta após o atendimento e que, portanto, não sofreram o desfecho cirurgia ou óbito e o grupo Caso, composto por pacientes que sofreram o desfecho, isto é, foram internados, foram submetidos a procedimento cirúrgico ou faleceram.
A análise estatística foi realizada por meio do software IBM SPSS Statistics Vesão 21.0 mediante construção de distribuições de frequências e de comparações entre as variáveis dependente e independente. Como medidas de tendência central foram utilizadas as medidas da média e desvio padrão e, também, da mediana e amplitude interquartil. O teste de Kolmogorov-Smirnov foi utilizado para determinar a normalidade ou não normalidade dos dados comparativos, sendo então empregados, respectivamente, os testes t de Student ou u de Mann-Whitney. Lançou-se mão, ainda, dos testes qui-quadrado de Pearson e razão de verossimilhança, com avaliação complementar da análise de resíduo para estes testes, e do teste $\mathrm{h}$ de Kruskal-Wallis, complementado pelo teste post-hoc de Dunn. Como medida de associação foi calculado odds ratio-OR-, com intervalo de confiança de 95\%, para as variáveis em que se identificaram resultados pertinentes s,9. $^{8,9}$.

\section{RESULTADOS}

Dos 330 atendimentos válidos avaliados, $235(71,2 \%)$ receberam alta após avaliação no serviço de pronto socorro. Os 95 (28,8\%) restantes alcançaram os desfechos primários deste estudo, sendo divididos em três subgrupos: a) internação hospitalar sem cirurgia, b) internação com cirurgia, c) óbito, conforme apresentado na figura 1. A amostra foi composta principalmente por mulheres $(64,8 \%)$ e a média de idade foi de $39,54( \pm 19,09)$ anos, variando entre quatro e 90 anos.

A comparação entre os grupos, detalhada na tabela 1, evidenciou significativa diferença estatística entre as médias de idade, frequência cardíaca, temperatura axilar, contagens leucocitária, de bastões, segmentados, e de níveis séricos de amilase, sendo que todos estes parâmetros apresentaram valores maiores em pacientes do grupo Caso. Esta constatação sugere pior desfecho nos pacientes que manifestam as referidas alterações. 


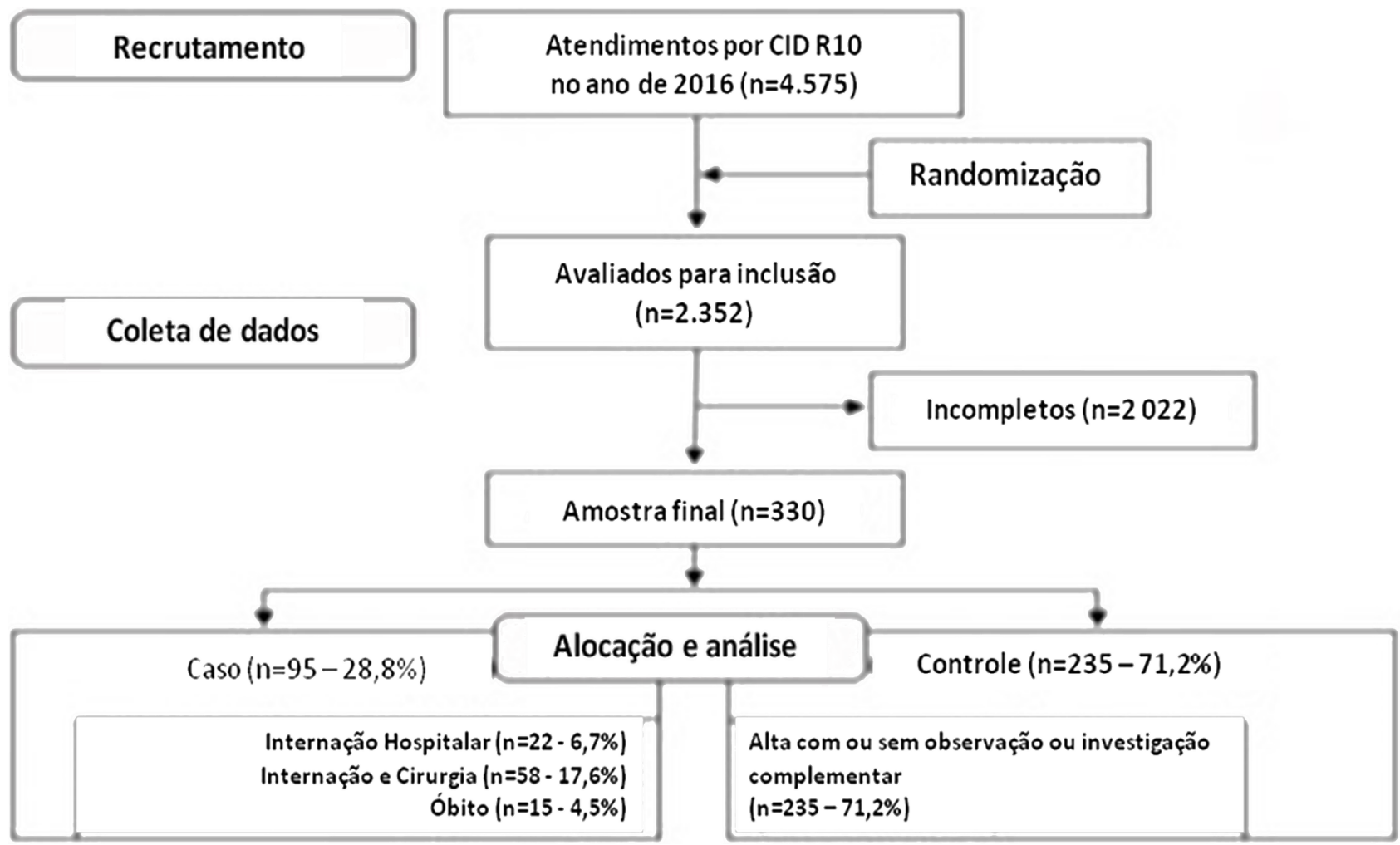

Figura 1. Esquema de etapas do estudo.

Tabela 1. Características dos grupos.

\begin{tabular}{|c|c|c|c|}
\hline Variáveis & Grupo Caso $n=95$ & Grupo Controle $n=235$ & Valor de $p$ \\
\hline Idade (anos)* & $44,40 \pm 19,32$ & $37,57 \pm 18,67$ & $0,004 t$ \\
\hline \multicolumn{4}{|l|}{$\operatorname{SexO} * *$} \\
\hline Feminino & $41(43,20)$ & $173(73,60)^{a}$ & $<0,001^{\text {ㄴ }}$ \\
\hline Masculino & $54(56,80)^{a}$ & $62(26,40)$ & \\
\hline \multicolumn{4}{|l|}{ Sinais vitais* } \\
\hline Frequência cardíaca & $90,52 \pm 20,66$ & $81,65 \pm 13,22$ & $<0,001 H$ \\
\hline Saturação periférica de oxigênio & $97,96 \pm 2,36$ & $98,49 \pm 1,37$ & $0,450^{+}$ \\
\hline Temperatura axilar & $37,13 \pm 0,95$ & $36,55 \pm 0,75$ & $<0,001^{t}$ \\
\hline Pressão arterial sistólica & $118,36 \pm 21,28$ & $126,40 \pm 17,80$ & $0,001^{\dagger}$ \\
\hline Pressão arterial diastólica & $70,66 \pm 14,12$ & $78,18 \pm 11,39$ & $<0,001^{+}$ \\
\hline \multicolumn{4}{|l|}{ Exames laboratoriais } \\
\hline Hematócrito* & $36,84 \pm 6,69$ & $39,31 \pm 3,89$ & $<0,001 H$ \\
\hline Hemoglobina* & $12,28 \pm 2,43$ & $13,23 \pm 1,50$ & $<0,001^{H}$ \\
\hline Leucócitos* & $12.812,13 \pm 6.010,86$ & $10.077,44 \pm 4.128,04$ & $<0,001^{+}$ \\
\hline Bastões*** & $214,00(133,0-340,5)$ & $170,00(109,0-221,5)$ & $<0,001^{\dagger}$ \\
\hline Segmentados*** & $8.394,00(4.867,5-12.359,0)$ & $5.678(4.190,0-8.160,5)$ & $<0,001+$ \\
\hline Linfócitos* & $2074,89 \pm 965,93$ & $2.395 \pm 997,19$ & $0,008+$ \\
\hline Amilase $^{* * *}$ & $65,00(48,0-86,0)$ & $56,00(46,0-75,5)$ & $0,026^{+}$ \\
\hline
\end{tabular}

* Valores expressos em média e desvio padrão; ** valores expressos em frequência e porcentagem; *** valores expressos em mediana e amplitude interquartil; + valor obtido por meio da aplicação do teste u de Mann-Whitney; " valor obtido por meio da aplicação do teste qui-quadrado de Pearson; + valor obtido por meio da aplicação do teste t-Student; a valores com significância estatística após análise de resíduo. 
Valores menores de pressão arterial sistólica e diastólica, de hematócrito, de hemoglobina e de linfócitos também foram estatisticamente associados ao grupo Caso. Os níveis de basófilos, eosinófilos e monócitos não demonstraram diferenças significativas entre os grupos, razão pela qual foram deliberadamente omitidos da tabela 1.

Quanto ao sexo, as evidências sugerem que pacientes do sexo masculino têm maior probabilidade estatística de pertencer ao grupo Caso em relação às pacientes do sexo feminino $(p<0,001)$. Não foram encontradas diferenças significativas entre os grupos quanto aos valores de saturação periférica de oxigênio $(p=0,450)$.

O exame parcial, ou qualitativo, de urina não apresentou diferença significativa entre os grupos no que tange à sedimentoscopia. A pesquisa de elementos anormais urinários, por outro lado, sugere que a presença de corpos cetônicos ( $p=0,022)$ ou de bilirrubina $(p=0,009)$ está mais estatisticamente associada ao grupo Caso em detrimento do grupo Controle.

No subgrupo de 22 (6,7\%) pacientes admitidos para internação hospitalar, sem que fosse necessária intervenção cirúrgica, obtiveramse 22 diagnósticos diferentes, como diverticulite, laceração hepática, cirrose, mesotelioma, suboclusão intestinal. Estas internações somadas totalizaram 82 dias de internação hospitalar, sendo a mais longa com 13 dias, para uma mediana de quatro dias.

O subgrupo de 58 (17,6\%) pacientes que foram internados e submetidos a procedimento cirúrgico, contabilizou 15 colecistectomias, nove laparotomias exploradoras, seis apendicectomias, seis desbridamentos ou drenagens de abcessos, cinco gastro ou duodenorrafias, cinco colectomias ou enterectomias, dentre outros menos frequentes. Este subgrupo acumulou 478 dias de internação hospitalar, com uma mediana de 4, variando entre 1, em alguns casos de colecistectomia e apendicectomia, e 70 dias de internação, em um caso de úlcera gástrica perfurada, tratada com gastrorrafia.
Os $15(4,5 \%)$ atendimentos que resultaram em óbito somaram juntos cerca de 100 dias de internação hospitalar. As causas dos óbitos foram: câncer de colo de útero $(n=3)$, câncer hepático $(n=2)$, trombose da veia porta $(n=1)$, sepse $(n=1)$ e politraumatismo $(n=1)$. Cânceres de bexiga, reto, pulmão, estômago e pâncreas também tiveram uma ocorrência cada. Dois óbitos não possuíram causa identificada.

A partir dos dados coletados, foi possível calcular o OR de algumas variáveis selecionadas, em relação aos grupos Caso e Controle. Pôde-se inferir que a chance de paciente com dor abdominal aguda e idade $\geq 50$ anos sofrer o desfecho é 2,67 vezes maior do que a chance de um paciente com idade inferior (IC95\%: 1,62-4,38). Sexo masculino, da mesma forma, apresenta maior chance de sofrer o desfecho, com OR de 3,67 (IC95\%: 2,23-6,05). Hematócrito abaixo de 35\% (OR=4,25; IC95\%: $2,33-7,77)$ e principalmente abaixo de $30 \%$ (OR=20,13; IC95\%: 4,47-90,51) também.

Leucocitose $\quad \geq 10.000 / \mathrm{mm}^{3} \quad(\mathrm{OR}=2,86$; IC95\%: 1,62-4,43) e, mais fortemente, $\geq 16.000 /$ $\mathrm{mm}^{3} \quad(\mathrm{OR}=6,26 ; \quad$ IC95\%: 3,11-12,62), tem significativamente mais chance de alocar o paciente no grupo Caso. Neutrófilos segmentados em número $\geq 7.700 / \mathrm{mm}^{3}$ apresentou OR de 2,62 (IC95\%: 1,60-4,28). Linfopenia $<2.000 / \mathrm{mm}^{3}$ encontrou OR de 1,70 (IC95\%: 1,05-2,76) a favor do grupo Caso.

Durante a análise exaustiva dos dados coletados no presente trabalho, os autores vislumbraram a possibilidade de extrapolar os padrões laboratoriais e clínicos encontrados através de um escore, concebido com base nos valores com diferenças estatisticamente significativas entre os grupos e subgrupos estudados, primariamente baseados em seus OR. Os pontos de corte de temperatura axilar, leucócitos e segmentados foram determinados com base na literatura ${ }^{10,11}$ e os demais, arbitrados após análise da curva ROC de cada teste ${ }^{8,9}$. 
Após atribuir uma pontuação para cada quesito, obteve-se um escore que variou de 0 a 12 pontos. Aos itens hematócrito e amilase, fez-se necessária pontuação graduada, a fim de melhorar as propriedades finais do escore. A figura 2 mostra as variáveis do escore e os grupos de risco, conforme pontuação no escore proposto de abdome agudo.

Os pontos de corte para os grupos de risco foram estabelecidos após análise estatística das médias de escore conforme o desfecho. Tal análise demonstrou médias sucessivamente maiores para os desfechos mais graves, conforme ilustra a figura 3.
O OR aplicado sobre o escore final demonstrou que pacientes com pontuação $=3$, ou seja, com risco diferente de baixo, têm 9,98 (IC95\%: 5,52-18,05) vezes mais chance de pertencer ao grupo Caso, em detrimento do grupo Controle.

\section{DISCUSSÃO}

As características demográficas da amostra em estudo encontram concordância com outros estudos semelhantes. A média de idade dos pacientes que procuram atendimento de urgência ou emergência é amplamente variável e pode divergir ainda mais quando analisadas as doenças específicas.

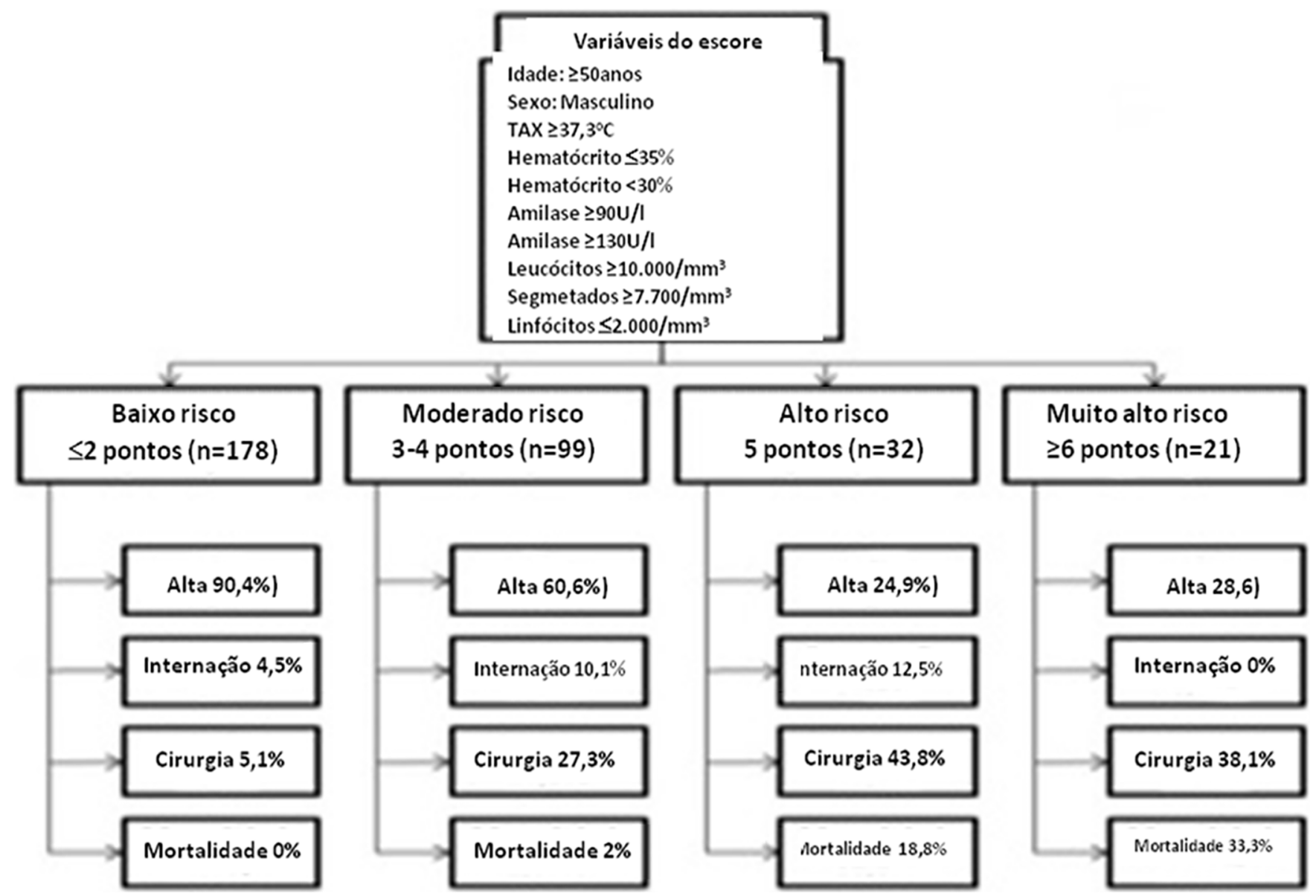

Figura 2. Escore de abdome agudo.

Na presença de uma das variáveis demonstradas na figura, o paciente deve receber 1 ponto. No caso da amilase, receberá 1 ponto se estiver $\geq 90$ U/l e mais 1 ponto se estiver $\geq 130 U / l$, totalizando o máximo de 2 pontos para o item amilase. Para o hematócrito, receberá 1 ponto se $\leq 35 \%$ e mais 1 ponto se estiver $\leq 30 \%$, totalizando o máximo de 2 pontos para o item hematócrito. Calculada a pontuação final, o paciente pode ser classificado em um dos quatro grupos de risco, conforme pontuação: baixo, com $0 \%$ de mortalidade; moderado, com 2\% de mortalidade; alto, com 18,8\% de mortalidade; e muito alto, com 33,3\% de mortalidade. TAX= temperatura axilar. 


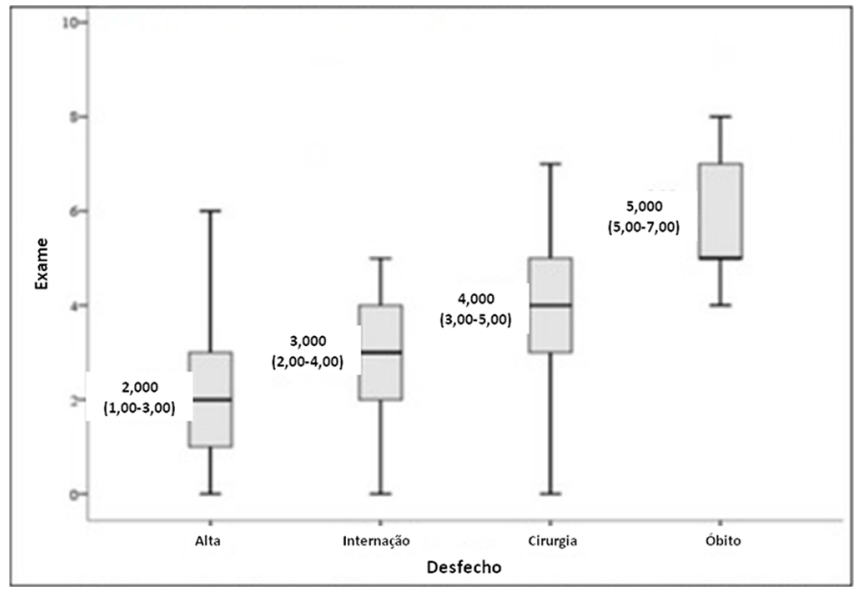

Figura 3. Pontuação mediana conforme desfecho. Mediana e amplitude interquartil do escore de abdome agudo, conforme o desfecho. O valor de p<0,001 foi obtido após realização do teste $h$ de Kruskal-Wallis, demonstrando significância estatística dos valores contidos na figura. O teste post-hoc de Dunn mostrou diferenças significativas nas comparações: Alta-Cirurgia ( $p<0,001) ;$ Alta-Óbito $(p=0,003)$; e não significativas nas comparações: AltaInternação $(p=0,056)$, Internação-Cirurgia $(p=0,406)$ e Cirurgia-Óbito $(p=0,083)$.

Na literatura, a média de idade parece ser de 25,3 anos para doenças como apendicite $\operatorname{aguda}^{10}$ e 51 anos para doenças como isquemia mesentérica aguda $^{12}$.

Em atendimentos de pronto socorro, a média de idade para todas as causas pode estar em torno de 46,7 anos ${ }^{12}$. Quando analisados os atendimentos por abdome agudo, a média etária de 39,54 anos encontrada neste estudo encontra sustentação em literaturas com metodologia semelhantes, sendo de 34 anos no estudo de Powers e Guertler ${ }^{5}$ e 39 anos no estudo do Acute Abdominal Pain (AAP) Study group ${ }^{13}$.

Quanto ao sexo, encontra-se 58\% de homens em estudos de apendicite ${ }^{10}$ e 69,8\% nos de isquemia mesentérica aguda ${ }^{14}$. Já, quando a queixa genérica de dor abdominal, a prevalência

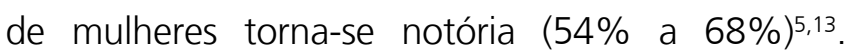
Estes dados validam os encontrados na presente série, com $64,8 \%$ de mulheres dentre a amostra total e 56,8\% de homens no grupo de Casos.

A taxa de internação hospitalar total no presente estudo foi de $28,8 \%$, o que é $10 \%$ superior à taxa encontrada por Powers e Guertler ${ }^{5}$, mas semelhante à taxa de $27,4 \%$ de um estudo realizado em 1972 na mesma instituição ${ }^{15}$. Dos 76 diagnósticos cirúrgicos identificados na série de Powers e Guertler ${ }^{5}, 36$ (47,3\%) foram colecistite, que também foi o diagnóstico mais frequente no presente estudo, com 19\% de ocorrência. Ainda, 4,3\% dos 1.166 pacientes internados faleceram, o que se assemelha, proporcionalmente, à taxa de $4,5 \%$ encontrada neste estudo. Dados do DATASUS ${ }^{7}$ sugerem letalidade de $9,62 \%$, dentre os pacientes internados com o CID10 R10.

O escore sugerido neste trabalho requisitou dos autores a arbitragem de alguns pontos de corte e a escolha de variáveis para sua composição. O ponto de corte da temperatura axilar que Alvarado ${ }^{10}$ estabeleceu em seu escore homônimo para o diagnóstico de apendicite aguda foi $\geq 37,3^{\circ} \mathrm{C}$, com sensibilidade de $73 \%$ e especificidade de $50 \%$. Outra análise ${ }^{16}$ encontrou sensibilidade de $70 \%$ e especificidade de $65 \%$, utilizando como ponto de corte, a temperatura $>37,7^{\circ} \mathrm{C}$.

Gans et al. ${ }^{2}$ avaliaram como baixa a sensibilidade (66\% a $78 \%$ ) de leucocitose $>10.000 /$ $\mathrm{mm}^{3}$ para a discriminação de causas urgentes de abdome agudo, definidas como aquelas que necessitam de tratamento em até 24 horas para prevenir complicações. A especificidade do mesmo teste foi determinada como $66 \%$.

O estudo de Alvarado $^{10}$ analisou retrospectivamente 277 prontuários de pacientes hospitalizados com suspeita de apendicite aguda. Destes, $89 \%$ tiveram o diagnóstico confirmado cirurgicamente. A partir destes dados, o autor determinou as propriedades estatísticas de achados clínicos e laboratoriais e encontrou variáveis que permitiram a construção de um escore que hoje leva seu nome, baseando-se na acurácia dos testes.

Ozkan et al. ${ }^{17}$ avaliaram as propriedades estatísticas do escore de Alvarado em uma amostra de 74 pacientes submetidos à apendicectomia, com média de idade de 36 anos e prevalência de homens (70,3\%). 
O escore apresentou sensibilidade de 54\%, especificidade de 73,3\%, valor preditivo positivo de $88,2 \%$, valor preditivo negativo de $29,7 \%$ e acurácia de 58\%. Comparativamente, a ultrassonografia apresentou, respectivamente, os valores $71,2 \%$, $47 \%, 82,2 \%, 31,8 \%$ e $66 \%$. É importante observar que as amostras de Alvarado e de Ozkan foram compostas por pacientes hospitalizados e já com diagnóstico de apendicite. É de se esperar que, nesta população, a prevalência de tal doença seja bastante elevada, o que favorece o desempenho do escore. Em contraponto, o presente estudo avaliou atendimentos realizados no setor de urgência e emergência cuja população é predominantemente composta por pacientes sem patologia cirúrgica e mesmo sem diagnóstico definido. Ainda assim, foram encontradas diferenças estatisticamente significativas entre os subgrupos e seus desfechos, o que sugere boa capacidade diagnóstica no escore proposto.

Mister reconhecer que os prontuários excluídos deste estudo, por estarem incompletos, possivelmente integrariam o grupo Controle. Por outro lado, sendo o critério de inclusão apenas o diagnóstico genérico de dor abdominal, representada pelo CID10 $\mathrm{R} 10$, pacientes que se apresentaram ao serviço médico e que receberam prontamente um diagnóstico específico ficaram além do alcance deste estudo. Tais pacientes integrariam o grupo Caso. Ademais, existe a possibilidade de alguns pacientes do grupo Controle terem retornado ao hospital e sofrido os desfechos fora do período de abrangência deste estudo. Certamente, tais alterações na amostra impactariam nas propriedades estatísticas do escore proposto.

Futuro estudo que utilize este escore de maneira prospectiva e cega, isto é, sem que o médico emergencista baseie-se em seu resultado para definir sua conduta, poderia esclarecer a utilidade e confirmar as propriedades do mesmo.
O mesmo estudo poderia avaliar a inclusão de elementos do exame físico, ou da anamnese, e de variáveis capazes de aumentar a sensibilidade do escore. Existem outros escores na literatura médica que objetivam o diagnóstico de patologias abdominais específicas. Os autores, entretanto, não identificaram nenhum cujo escopo fosse a classificação de risco de pacientes com dor abdominal de forma sindrômica, isto é, independente do diagnóstico. Tal fato constitui possivelmente a maior virtude do presente trabalho, pois, diante disso, poderse-ia considerar a utilização do escore de abdome agudo como auxiliar na decisão de necessidade de hospitalização.

O`Brien ${ }^{6}$ considera como critério para internação hospitalar a presença de sinais ou sintomas de gravidade em pacientes de alto risco. Outros autores $^{18}$ consideraram como patologia grave aquela em que coexistiu febre com quaisquer dos seguintes itens: necessidade de antibioticoterapia endovenosa, uso de fármacos vasoativos endovenosos, cirurgia, drenagem radiológica, internação em unidade de terapia intensiva, alterações da série branca, da série vermelha ou da contagem de plaquetas.

Mediante uso do escore proposto neste trabalho, a possibilidade de internação hospitalar poderia ser considerada nos casos em que o escore apresente um resultado distinto de baixo risco (pontuação 3) ou, principalmente, alto ou muito alto risco (pontuação 5). Os autores ressalvam, por fim, que ainda que tenham incessantemente buscado anular todos os vieses possíveis, seja na etapa de coleta ou de análise dos dados, não é possível excluir a ocorrência de viés de aferição primário, visto que todos os dados, mas principalmente os sinais vitais, como é o caso da temperatura axilar, foram coletados e anotados sem supervisão metodológica. 
O presente estudo encontrou variáveis estatisticamente associadas aos desfechos analisados, o que ajuda a determinar o perfil de pacientes mais sujeitos à internação hospitalar, cirurgia ou óbito. Os autores desenvolveram um sistema de escore que demonstra capacidade de estratificar os pacientes com dor abdominal conforme a chance de desfecho, baseando-se apenas nos parâmetros de idade, sexo, temperatura axilar, hematócrito, leucograma e amilase sérica. Esta ferramenta, passível de lapidação, pode ser aprimorada, através de novos estudos, até o ponto de distinguir correta e precisamente os pacientes sem doença daqueles com risco de óbito. Por hora, ficou evidente para os autores que o escore proposto possui boa capacidade para identificar pacientes com maior chance de óbito.

\title{
A B S T R A C T
}

\begin{abstract}
Objective: to establish a correlation between objective data collected at the initial evaluation and the outcomes in cases of acute abdominal pain. Methods: we conducted a retrospective, case-control study in which we reviewed medical records of patients treated at an emergency room in 2016, diagnosed with abdominal and pelvic pain. Results: of the 2,352 medical records evaluated, we considered 330 valid for the study. Of these patients, 235 (71.2\%) were discharged and the remaining 95 (28.8\%) were hospitalized, submitted to surgery, or died. The statistical analysis demonstrated that male gender, age $\geq 50$, axillary temperature $>37.3^{\circ} \mathrm{C}$, anemia, leukocytosis $>10,000 / \mathrm{mm}^{3}$, neutrophil count above $7,700 / \mathrm{mm}^{3}$, lymphocyte count less than $2,000 / \mathrm{mm}^{3}$ and hyperamylasemia were variables in independently associated with worse outcome. Conclusion: the presence of three or more of the evaluated variables greatly increases the chances of a patient suffering the outcomes of surgery or death, the chance of death being greater the greater the number of variables present.
\end{abstract}

Keywords: Abdominal Pain. Abdomen. Acute. Propensity Score. Surgery Department. Hospital.

\section{REFERÊNCIAS}

1. Squires RA, Portier RG. Abdome agudo. In: Townsend Jr. CM, Beauchamp RD, Evers BM, Mattox KL, editores. Sabiston tratado de cirurgia: a base biológica da prática cirúrgica moderna. 19a ed. Rio de Janeiro: Elsevier; 2015. p. 1141-59.

2. Gans SL, Pols MA, Stoker J, Boermeester MA; expert steering group. Guideline for the diagnostic pathway in patients with acute abdominal pain. Dig Surg. 2015;32(1):23-31.

3. Feres $\mathrm{O}$, Parra RS. Abdômen agudo. Medicina (Ribeirão Preto). 2008;41(4):430-6.

4. Meneghelli UG. Elementos para o diagnóstico do abdômen agudo. Medicina (Ribeirão Preto). 2003;36(2/4):283-93.

5. Powers RD, Guertler AT. Abdominal pain in the ED: stability and change over 20 years. Am J Emerg Med. 1995;13(3):301-3.

6. O'Brien MC. Acute abdominal pain. In: Tintinalli JE, Stapczynski JS, Ma OJ, Cline DM, Cydulka RK, Meckler GD; The American College of Emergency Physicians. Tintinalli's emergency medicine: a comprehensive study guide. 7th ed. New York: McGraw-Hill; 2016. p. 481-9.
7. Brasil. Ministério da Saúde. Sistema de Informações Hospitalares do SUS (SIH/SUS)/DATASUS. Morbidade Hospitalar do SUS: CID-10 R10, Período Jan/2018.

8. Oliveira Filho PF. Epidemiologia e bioestatística: fundamentos para a leitura crítica. 1a ed. Rio de Janeiro: Rubio; 2015.

9. Medronho RA, Bloch KV, Luiz RB, Werneck GL. Epidemiologia. 2a ed. São Paulo: Atheneu; 2009.

10. Alvarado A. A practical score for the early diagnosis of acute appendicitis. Ann Emerg Med. 1986;15(5):557-64.

11. Kabir SA, Kabir SI, Sun R, Jafferbhoy S, Karim A. How to diagnose an acutely inflamed appendix; a systematic review of the latest evidence. Int J Surg. 2017;40:155-62.

12. Barreto RF, Gomes CZL, da Silva RM, Signorelli AAF, Oliveira LF, Cavellani CL, et al. Avaliação de dor e do perfil epidemiológico, de pacientes atendidos no pronto-socorro de um hospital universitário. Rev Dor. 2012;13(3):213-9.

13. Acute Abdominal Pain (AAP) Study group. Diagnostic accuracy of surgeons and trainees in assessment of patients with acute abdominal pain. Br J Surg. 2016;103(10):1343-9. 
14. Andersson RE, Hugander AP, Ghazi SH, Ravn $\mathrm{H}$, Offenbartl SK, Nyström PO, et al. Diagnostic value of disease history, clinical presentation, and inflammatory parameters of appendicitis. World J Surg. 1999;23(2):133-40.

15. Brewer RJ, Golden GT, Hitch DC, Rudolf $L E$, Wangensteen SL. Abdominal pain. An analysis of 1,000 consecutive cases in a University Hospital emergency room. Am J Surg. 1976;131(2):219-23.

16. Wang Z, Chen JQ, Liu J, Tian L. A novel scoring system for diagnosing acute mesenteric ischemia in the emergency ward. World J Surg. 2017;41(8):1966-74.

17. Ozkan S, Duman A, Durukan P, Yildirim A, Ozbakan O. The accuracy rate of Alvarado score, ultrasonography, and computerized tomography scan in the diagnosis of acute appendicitis in our center. Niger J Clin Prac. 2014;17(4):413-8.
18. San Martín López JV, Casas Rojo JM, Mateo Rodríguez O, Frutos Pérez B, Farfán Sedano A, Cabello Clotet $N$, et al. Fiebre en urgencias: detección de patología grave. Rev Clin Esp. 2008;208(3):130-4.

Recebido em: 29/06/2019

Aceito para publicação em: 11/09/2019

Conflito de interesse: nenhum.

Fonte de financiamento: nenhuma.

\section{Endereço para correspondência:}

Felipe Antônio Cacciatori

E-mail: felipe_cacciatori@hotmail.com

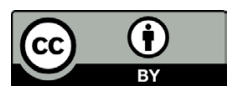

